\title{
Efficacy and safety of phytoestrogens in the treatment of postmenopausal depressive disorders: a systematic review and meta-analysis
}

\author{
Li Jieyun ${ }^{1}$, Huijuan $\mathrm{LI}^{2}$, Yan Peijing ${ }^{3}$, Liping Guo ${ }^{4}$, Jingwen $\mathrm{Li}^{4}$, Jiani $\mathrm{Han}^{5}$, Jie Qiu ${ }^{6}$, and \\ Kehu Yang ${ }^{7}$ \\ ${ }^{1}$ Evidence-Based Medicine Center, School of Basic Medical Sciences, Lanzhou University, \\ Lanzhou, PR China \\ ${ }^{2}$ Taiyuan Central Hospital, Taiyuan, PR China \\ ${ }^{3}$ Department of Epidemiology and Health Statistics, West China School of Public Health \\ and West China Fourth Hospital, Sichuan University \\ ${ }^{4}$ Key Laboratory of Evidence Based Medicine and Knowledge Translation of Gansu \\ Province \\ ${ }^{5}$ Evidence-Based Medicine Center, School of Basic Medical Sciences, Lanzhou University \\ ${ }^{6}$ Gansu Provincial Maternity and Child Care Hospital \\ ${ }^{7}$ Evidence Based Medicine Center
}

December 21, 2020

\begin{abstract}
Background:Depression is one of the most common and specific symptoms among postmenopausal women, leading to significant personal, family, and economic burdens. Some studies have shown that phytoestrogens can help relieve symptoms of depression. Objectives: This systematic review and meta-analysis aims to assess the efficacy and safety of phytoestrogens in treating depression among postmenopausal women. Methods: A comprehensive search for relevant studies published until November 25, 2020, was conducted in PubMed, the Cochrane Library, Chinese Biomedical Literature Database, Web of Science, and EMBASE. Statistical analyses were performed with Stata12.0, Comprehensive Meta-Analysis 3.0, and Review Manager 5.4. Results: 2,183 studies were identified and 10 studies were included, involving 1,248 participants. The quality of the four studies was assessed as high risk, six studies were assessed as unclear. The analyses conducted according random effects model indicated a positive effect on depressive symptoms for postmenopausal women compared to the placebo ( $\mathrm{SMD}=-0.45 ; 95 \%$ [CI] $=-0.60$ to $-0.29 ; \mathrm{Q}=40.42, \mathrm{df}=18, \mathrm{P}=0.002 ; \mathrm{I} 2=55.5 \%$ ). The high dose phytoestrogens (dose $>100 \mathrm{mg} /$ day) have better effectiveness (SMD $=-0.48 ; 95 \%[\mathrm{CI}]=-0.76$ to $-0.20 ; \mathrm{I} 2=52.8 \%, \mathrm{P}<0.05)$ than low dose $(25 \mathrm{mg} /$ day[?]dose[?]100mg $/$ day $)(\mathrm{SMD}=-0.45 ; 95 \%[\mathrm{CI}]=-$ 0.68 to- $0.23 ; \mathrm{I} 2=66.7 \% ; \mathrm{P}>0.05)$ and ultralow dose $(00.05)$. Isoflavones had better effectiveness $(\mathrm{SMD}=-0.52 ; 95 \%[\mathrm{CI}]=-0.75$ to $0.30 ; \mathrm{I} 2=65.8 \%, \mathrm{P}<0.05)$ than lignans of phytoestrogens $(\mathrm{SMD}=-0.23 ; 95 \%[\mathrm{CI}]=-0.05$ to $0.01 ; \mathrm{I} 2=0 \%, \mathrm{P}>0.05)$. The effectiveness varies in regions. The adverse reactions frequently reported were gastrointestinal symptoms and cold or upper respiratory tract infection. Conclusions: Phytoestrogen can relieve depression symptoms among postmenopausal women. Highdose isoflavones is the most effective. Although mild adverse reactions have been reported, phytoestrogen could be considered as a complementary treatment for postmenopausal depression.
\end{abstract}

\section{Introduction}

Menopause is generally defined as the "cessation" of menstruation for 12 consecutive months and is considered as the end of a woman's reproductive life $^{1}$. During this period, women always experience many metals 
distresses, such as poor concentration, agitation, anxiety, depression, and difficulty controlling themselves ${ }^{2,3}$. Depression was one of the most common and specific symptoms of menopause ${ }^{4-6}$, which may cause some serious problems such as disability and death ${ }^{7,8}$, leading to significant individual, family, and society burdens ${ }^{9}$.

Estrogen is the most used to treat depression and for relieving other symptoms among postmenopausal women in clinical ${ }^{10,11}$. However, several side effects and risks have been reported after estrogen treatment, including stroke, thromboembolism events, breast cancer, and vascular diseases ${ }^{12}$. Furthermore, Morrison et $a l$. reported that hormone replacement therapy (HRT) does not treat post-menopause including relieving depressive symptoms in them ${ }^{13}$.

Nowadays, women are preferring to choose treatments with herbal medicine rather than exposing themselves to the risks associated with $\mathrm{HRT}^{3,14}$. Researchers have proposed that phytoestrogens, which are compounds extracted from plants ${ }^{15}$ can be used as an alternative approach to treating post-menopausal depression, without the risks associated with taking estrogens ${ }^{16}$. There are two main types of phytoestrogens: isoflavones and lignans. Isoflavones are found mainly in beans, fruits, and vegetables, especially soybeans; Lignans are found in lentils, cereals, wheat, and black rice, as well as fennel, sunflower seeds, and onions ${ }^{17}$.

The efficacy of phytoestrogens remains controversial. Some research findings have indicated a significant improvement in relieving depression among post-menopausal ${ }^{18,19}$, while other studies have argued that the treatment of soy isoflavones does not reduce depression ${ }^{20}$. There has been a meta-analysis published in 2018 , which included 3 studies and 316 participants (149 in the phytoestrogen group and 167 in the placebo group) that showed phytoestrogen consumption is associated with a significant reduction in depressive symptoms among postmenopausal women ${ }^{21}$. However, as only three articles included all published in 2009 and 2010, more than twice as many eligible studies ranging from 2002 to 2017 were ignored. There are no convincing conclusions regarding the effects of phytoestrogens on postmenopausal depression until now.

The present systematic review and meta-analysis of randomized controlled trials (RCTs) aim to investigate the efficacy and safety, as well as the influencing factor of phytoestrogens in alleviating depression among postmenopausal women.

\section{Method}

The protocol for this systematic review has been registered on the International prospective register of systematic reviews (PROSPERO No. CRD4201804493). The Cochrane Handbook for Systematic Reviews of Interventions and PRISMA Statement helped guide the conduct and reporting of this review ${ }^{22}$.

\subsection{Inclusion and exclusion criteria}

Inclusion criteria were as follows: (1) Enrolled women with at least 12 months of menopause at baseline, and without the physical disease; (2) women experienced postmenopausal depression and did not undergo hormone therapy; (3) were treated with an oral phytoestrogen; (4) reported data that can be used to calculate the effectiveness; (5) the study was an RCT.

Studies were excluded due to the following reasons:(1) reported previous or current use of hormone therapy, (2) history of ovariectomy or hysterectomy, or no menopausal symptoms; (3) numerical outcome data was not provided, (4) abstract of meeting or letters. The degree of mental disorders was not considered among the inclusion or exclusion criteria.

\subsection{Data sources and search strategy}

A comprehensive search was conducted for relevant studies that were published in English or Chinese, in databases including PubMed, the Cochrane Library, Chinese Biomedical Literature Database, Web of Science, and EMBASE on November 25, 2020. The search keywords included (depression OR Depress* OR blue* OR melancho*) AND ("Plant Estrogens" OR "Phyto-Estrogen" OR "Estrogen-Like Plant Extracts") AND (postmenopausal* OR menopause* OR "postmenopausal period" OR involutional)]. Details on the search strategy have been provided in Supplemental Material Table 1. The reference lists of the studies were searched manually to identify additional studies ${ }^{23}$. The Clinical Trials.gov website was also searched for 
RCTs that were registered as completed but not yet published ${ }^{24}$. A flow diagram of the systematic search and study selection process is shown in Figure 1.

\subsection{Selection of data extraction}

Eligible studies were screened in their entirety and developed a data extraction form, and the information including authors and year of publication, publication type, sample size, gender, type of treatment was recorded in an Excel spreadsheet. We pre-tested it on five studies and subsequently adapted the final version. If the inclusion criteria were met, the full-text of each study was coded by the first authors (J.Y. and H.J) using this template, difficulties in deciding the inclusion status of studies were discussed between two authors and resolved by consensus.

If there was any evidence for the use of the same sample in different publications, authors were contacted for clarification $^{25}$. If it was confirmed that two studies were based on the same data, we chose the study that reported the most comprehensive results. Study authors were also contacted to request additional information if a study was eligible based on the inclusion criteria, but not all required data could be retrieved from the full-text. Overall, we adopted a conservative procedure in that we excluded studies for which ambiguity about the primary data source could not be resolved or if the information necessary for effect size calculation could not be acquired ${ }^{26}$. If studies reported on the results of two independent treatment subgroups, effect sizes for each subgroup were included in the meta-analysis and treated as a separate study to enable moderator analyses for the type of treatment.

\subsection{Heterogeneity, sensitivity, and publication bias}

Based on the Cochrane Handbook Version 6.1.0, 2020, heterogeneity was assessed using Cochrane's Qstatistics to estimated the standard deviation of the true effect sizes and the $I^{2}$-Index ${ }^{27}$. A significant Q-test indicates that effect sizes of primary studies do not belong to the same distribution of effect sizes. When performance qualification statistics [?]0.05 ( $P$ [?]0.05), was considered no significant heterogeneity among the included studies ${ }^{25} . I^{2}$ index is used to the estimated amount of variability in the true effect sizes, and the proportion of observed variability that can be explained by true heterogeneity. Values of $25 \%, 50 \%$, and $75 \%$ for the $I^{2}$-index indicate low, moderate, and high degrees of heterogeneity, respectively. To further explain the heterogeneity of our main results.

The "Leave-one-out" method is used in sensitivity analyses to check for outliers that potentially influence the results of the meta-analysis disproportionately ${ }^{25}$. All analyses were performed repeatedly with each study removed once to detect whether overall results depend on a single study.

Publication bias means that statistically significant results are more likely to be published, while statistically insignificant results are less likely to be published. Therefore, these studies with no significant significance could be more likely to remain in the "file drawer" ${ }^{28}$. Publication bias was assessed by three methods. Funnel plots illustrate the effect sizes of primary studies as a function of study precision, operationalized by the standard errors of studies. Asymmetry in plots can indicate publication bias ${ }^{29}$. Egger's regression test yields a statistical verification of funnel plot asymmetry. If any bias could be assumed based on these analyses, we planned to apply the trim-and-fill procedure to estimate the unbiased overall effect ${ }^{30}$.

\subsection{Risk of bias}

The Risk of Bias 2.0 (RoB 2,0) tool in Review Manager 5.4 software is used to evaluate the risk of bias ${ }^{27}$. According to sequence generation, allocation concealment, blinding of outcome assessors, incomplete outcome data, selective outcome reporting, and other sources of bias, each study was determined as having a low, high, or unclear risk of bias $^{27}$. These assessments were conducted under the guidance of the Cochrane Handbook for Systematic Reviews of Interventions (Version 6.1.0) and the study was conducted according to the Preferred Reporting Items for Systematic reviews and Meta-Analyses guidelines. Two authors (J.W and L.P) independently assessed the identified studies, the conflicts were resolved by discussion. 


\subsection{Data synthesis and statistical analysis}

The fixed-effects model and the random-effects model are based on different assumptions. The results of meta-analysis using fixed-effect models are limited to specific populations. As we cannot expect these studies included in the research to show the same widespread impact; the fact that the studies were conducted under different conditions (i.e., geography, dosage, measurement, etc.) could indispensably cause differences among the results. Thus, in the identification of effect sizes during the present meta-analytical processes, analyses were conducted according to the random effects model ${ }^{31}$. We computed standardized mean differences (SMD) with $95 \%$ confidence intervals $(95 \% \mathrm{CI})^{27}$ to estimate effect sizes for continuous outcomes. Besides, we use stratified analysis to explore subgroup analysis. Data were further analyzed by using Stata 12.0, Comprehensive Meta-Analysis 3.0, and Review Manager 5.4.

\section{Results}

\subsection{Selection and Characteristics of Studies}

Of 2,183 studies related to postmenopausal depression that were identified, 44 were selected for full-text review, and 10 studies with 19 independent reports were included in the meta-analysis (see Fig.1) 16,18,20,32-38.

These studies were published across eight countries, including Japan, Korea, India, Brazil, Canada, Austria, Australia, and Italy between 2002 and 2017, totaling 1,248 participants. Six scales were used to evaluate the depression index before and after treatment, including the center for epidemiological studies depression scale $(\mathrm{CED}-\mathrm{S})^{7}$, the Hamilton depression rating scale (HADS $)^{39}$, the Zung's self-rating depression scale $(\mathrm{SDS})^{40}$, the profile of mood states (POMS) ${ }^{41}$, the Greene postmenopausal scale (GSC) ${ }^{42}$ and the Kupperman menopause index (KMI) ${ }^{43}$. All the included studies in this meta-analysis used the evaluation scales to evaluate depression in postmenopausal women. Six studies were by clinician rating, three studies were by self-rating, one study remained was assessed by both clinician and self-rating. Characteristics of the studies are shown in Table 1.

\subsection{Risk of Bias}

The quality of the four studies was assessed as high risk, six studies were assessed as unclear. Participants were selected, and both the baseline and follow-up conditions are well described. Random sequence generation was deemed to be adequate in five of the 10 trials $^{16,18,35-37}$. Blinding of the outcome assessment is unknown across five trials ${ }^{20,32,33,37,38}$. One trial ${ }^{33}$ helped assess the high risk of bias in the selective outcome since the pre-specified primary outcomes were not reported. Three ${ }^{33,36,37}$ of the studies reported conflicts of interest. The risk of bias assessment is indicated in Figure 2a, b.

\subsection{Efficacy of phytoestrogens}

A total of 10 studies with 19 independent reports were included in this meta-analysis, with 1,248 participants. The results indicated that phytoestrogens had a positive effect compared to placebo on depressive symptoms for postmenopausal women $\left(\mathrm{SMD}=-0.45 ; 95 \%[\mathrm{CI}]=-0.60\right.$ to $-0.29 ; \mathrm{Q}=40.42, \mathrm{df}=18, P=0.002 ; I^{2}=55.5 \%$ ) (Fig.3).

\subsection{Sensitivity and Publication Bias}

According to the "leave-one-out" strategy, 19 outcomes estimated values from - 0.479 to -0.411 were got, indicated that there were no particularly prominent sensitivity issues in the included literature (Supplementary Material Fig 1).

The shape of the funnel plots appeared symmetrical in the comparison model showed that most effect sizes seem to locate symmetrically upwards the graph, and scatter around both sides of the line. Egger's regression test did not show a publication bias $(P=0.939)$. Besides, there was no obvious change in the results after the trim and -fill estimate. (Supplementary Material Fig.2)

\subsection{Typeof phytoestrogens}


A total of 10 studies with 19 independent reports with 1,248 participants were included in investigating the effects of different phytoestrogens on depressive symptoms in postmenopausal women. Among them, six studies employed isoflavones including red clover isoflavones ${ }^{18,37}$, daidzein isoflavone ${ }^{32}$, soy isoflavones ${ }^{20}$, isoflavones aglycone ${ }^{33}$ and phytoestrogen genistein ${ }^{16}$; two studies employed lignans including Schisandra chinensis $^{34}$ and Kava-Kava ${ }^{38}$; two studies employed fenugreek extract ${ }^{35}$ and resveratrol ${ }^{36}$ which could not give a clear classification. The effectiveness of postmenopausal women based on two different categories of phytoestrogens indicated that isoflavone $\left(\mathrm{SMD}=-0.52 ; 95 \%[\mathrm{CI}]=-0.75\right.$ to $\left.0.30 ; I^{2}=65.8 \%, P<0.05\right)$ was better than treatment with lignans $\left(\mathrm{SMD}=-0.23 ; 95 \%[\mathrm{CI}]=-0.05\right.$ to $\left.0.01 ; I^{2}=0 \% ; P>0.05\right)$ (Supplementary Material Fig.3).

\subsection{Dose of phytoestrogens}

To compare the effects of various doses of phytoestrogens more intuitively, phytoestrogens intake dose were classified $^{33}$. After sorting by the daily dosage of phytoestrogens intake. Doses more than 0 and less than $25 \mathrm{mg}$ were classified as the ultra-low dose group (Ultra-low dose: $0<$ dose $<25 \mathrm{mg} /$ day), doses more or equal to $25 \mathrm{mg}$ and less or equal to $100 \mathrm{mg}$ were in the low dose group (Low Dose: $25 \mathrm{mg} /$ day[?]dose[?]100mg/day), while doses more than $100 \mathrm{mg}$ were in the high dose group (High Dose: dose $>100 \mathrm{mg}$ ). According to the various dosages in Supplementary Material Figure 4, the results indicated that compared with the low dose $\left(25 \mathrm{mg} /\right.$ day[?]dose[?]100mg/day) $\left(\mathrm{SMD}=-0.45 ; 95 \%[\mathrm{CI}]=-0.68\right.$ to- $\left.0.23 ; I^{2}=66.7 \% ; P>0.05\right)$ and ultra-lowdose group $\left(0<\right.$ dose $<25 \mathrm{mg}$ ) ( $\mathrm{SMD}=-0.31 ; 95 \% \mathrm{CI}=-0.47$ to $\left.0.04 ; I^{2}=0 \%, P>0.05\right)$, the intake of phytoestrogens in the high dose group (dose $>100 \mathrm{mg} /$ day) was the most effective in improving the symptoms of menopausal depression $\left(\mathrm{SMD}=-0.48 ; 95 \%[\mathrm{CI}]=-0.76\right.$ to $\left.-0.20 ; I^{2}=52.8 \%, P<0.05\right)$.

\subsection{Measurements of depression}

Ten RCTs with 19 independent reports and 1,248 participants were included in investigating the effects of different measurements on depressive symptoms in postmenopausal women. All the included studies in this meta-analysis used the evaluation scales to evaluate depression in postmenopausal women. The result based on different assessment scales were detected in Supplementary Material Figure 5, indicated that using the HDS as an assessment scale was associated with the most varied data $(\mathrm{SMD}=-0.65 ; 95 \%[\mathrm{CI}]=-1.38$ to $\left.0.08 ; I^{2}=80.3 \% ; P<0.05\right)$. Meanwhile, the data in GCS $\left(\mathrm{SMD}=-0.37 ; 95 \%[\mathrm{CI}]=-0.62\right.$ to $-0.21 ; I^{2}=54.1 \%$; $P<0.05)$ and SDS (SMD $=-0.59 ; 95 \%[\mathrm{CI}]=-0.92$ to $\left.0.25, I^{2}=58.4 \% ; P>0.05\right)$

To explore whether different evaluation scales could reduce the moderate and high heterogeneity, assessment scales of specific postmenopausal evaluation $\left(\mathrm{GCS}^{42}\right.$ and $\left.\mathrm{KMI}^{43}\right)$ with depression items were excluded for supplementary analysis. Results showed that heterogeneity of the isoflavone group slightly decreased from $65.8 \%$ to $60.6 \%$ ( $P=0.01$ ) (Supplementary Material Fig.6), as well as the heterogeneity of the low dose group obviously decreased from $66.7 \%$ to $10.5 \%(P=0.34)$ (Supplementary Material Fig.7).

\subsection{Regions of treatment}

Included ten studies with 19 independent reports from eight countries were divided into five continents, to explore whether post-menopausal women who took phytoestrogens had different rates of depression relief. We found that the postmenopausal women in Europe had the best depressive remission among the five continents $\left(\mathrm{SMD}=-0.60,95 \%[\mathrm{CI}]=-0.85\right.$ to $\left.-0.34 I^{2}=54 \%, P<0.05\right)$, better than the women in other regions (In Supplementary Material Fig.8),

\subsection{Adverse Events}

106 participants have reported 17 different adverse reactions in five of the ten included studies ${ }^{20,32,34,37,38}$. We classified all reported adverse reactions into three categories: gastrointestinal symptoms, physical pain, and other adverse events (Table 2).

Gastrointestinal symptoms. Of the four studies that included 253 participants, 38 reported adverse reactions to gastrointestinal symptoms. Among them, 20 participants reported abdominal pain in four studies ${ }^{20,32,34}$; five participants reported nausea in two studies; two participants reported constipation in 
one study ${ }^{20}$; one participant increased in appetite, seven reported diarrheas in two studies ${ }^{18,20}$; one reported an epigastric problem, and one reported flatulence in one study ${ }^{20}$.

Physical pains. Of the three studies that included 221 participants, 43 reported adverse reactions to physical pain ${ }^{18,20,34}$. Based on the study research on red clover isoflavones by Lipovac et.al. $(2010)^{18}$, nine participants reported headache, 18 participants reported myalgia and 12 participants reported Arthralgia. In the study of soy isoflavones by Rilvaet. al. (2009), two participants reported headache and one reported pain breast. One participant reported headache with Schisandra Chinensis treatment in Park et.al. $(2016)^{34}$.

Other events. Of the three studies that included 221 participants, 23 reported adverse reactions to other events. In the study of red clover isoflavone researched by Lipovac et.al. (2010) ${ }^{18}, 19$ participants reported cold or upper respiratory tract ${ }^{18}$. One participant reported tremor of extremities, insomnia, and generalized rash in Cagnacci et.al. $(2003)^{20}$. One participant reported infection with daidzein isoflavone in Ishiwata et.al. $(2009)^{32}$.

\section{Discussion}

Our study aims to investigate the efficacy and safety, as well as the influencing factor of phytoestrogens in alleviating depression among postmenopausal women. The results indicated that phytoestrogens have a positive significant effect at reducing depression among postmenopausal women, and would be more effective when using high dosages (High Dose: dose $>100 \mathrm{mg}$ ). Lephart et al ${ }^{44}$ has published research in 2005 on the mechanism of phytoestrogen action, which investigated the levels of brain Bcl-2-associated death protein (a proapoptotic member of Bcl-2 protein family) and neuron-specific $\beta$-III tubulin (an early marker of neuronal differentiation/survival) in rats, suggesting that soy isoflavones can potentially ameliorate anxiety and quicken recovery from trauma. Therefore, based on animal trials, the effectiveness of phytoestrogens has scientifically been proved and they can be used to relieve mental disorders.

The effects of the isoflavone group were better than the lignans group. Among them, the red clover isoflavones indicated the best effectiveness among all included phytoestrogens (SMD=-1.05; 95\% [CI] $]=-1.45$ to -0.65 ). As one study found in legumes, red clover isoflavone through estrogen receptor (ER) binding and activation of ER-dependent gene transcription, which has a relatively high binding affinity for the ER- $\alpha$ and ER- $\beta$, isoflavones demonstrate up to $\sim 1600$ times lower affinity for ER- $\alpha^{45}$. This may explain the significant effect of isoflavones.

High doses (dose $>100 \mathrm{mg} /$ day) of isoflavone phytoestrogens are more effective than low doses. The reason why isoflavone at the higher dose effectively alleviated menopausal symptoms in the current study may be because the isoflavones were completely presented in the aglycone form, which is absorbed faster and in greater amounts than the glucoside form ${ }^{46}$. Other studies reported excellent tolerance for the use of soy isoflavones to extract in the daily dose from 40 to $50 \mathrm{mg}^{47,48}$. However, the alarm raised by the Italian study that showed an increase in endometrial hyperplasia after a 5 -year intake of $150 \mathrm{mg} /$ day of isoflavones ${ }^{33}$. Therefore, to identify the best dose to use, which could better alleviate the symptoms of depression in postmenopausal women, we look forward to more primary studies on phytoestrogens in the future.

The postmenopausal women in Europe had the best depressive remission among the five continents. As the dietary habits of the women in various countries are different, such as the preferable diet of having soy products in Japan and Korea may cause an effect on the results of the phytoestrogens ${ }^{49}$. Additionally, considering the ethnic diversity in different regions, more experiments are needed to explore the specific conclusions.

106 participants have reported 17 different adverse reactions in five of the ten included studies with 379 participants. Mild intensity abdominal pain is the predominant AE of phytoestrogens in postmenopausal women with depression. However, concerning the adverse events searched in the included studies, due to the sporadic nature of reported by various groups, it was not possible to determine the association of these events with the use of phytoestrogens. 
Although this systematic review and meta-analysis found that phytoestrogens were effective in postmenopausal women, our outcomes need to be treated with caution due to several limitations. Firstly, the number of studies included in this meta-analysis was not very many that some subgroup analyses could only be combined with two or three studies. Besides, though all the studies we included were RCTs, there was the weakness of methodologies in several studies, such as inadequate treatment allocation, small sample size, and unclear blinding method. Secondly, the sensitivity and specificity of various measurements are all different, and there is no utilized gold-standard diagnostic for depression. Hence, we hope that specific depression scales can be unified in the evaluating methods in future clinical research. Thirdly, even we used subgroup analysis to explore the sources of heterogeneity, but the results still showed high heterogeneity. Therefore, more caution is needed in the promotion of the results ${ }^{50}$. There still needs to be more clinical trials on different phytoestrogens, to identify the most appropriate dosage, duration, and other treatment applications to relieve menopausal depression as much as possible.

\section{Conclusion}

A favorable effect of phytoestrogens indicated can help manage depression among postmenopausal women, especially with a high dose of isoflavones. The adverse effects of phytoestrogens on postmenopausal women are not yet clear and more relative high-quality RCT studies are needed in the future that can help provide more supportive evidence.

\section{Contributors}

YKH designed the study.

LJY and LHJ did the literature searches and designed the data extraction form.

LJW and GLP extracted data.

LJY and LJW cross-checked data extraction.

LJY and HJN did the statistical analyses.

QJ and YPJ supervised the statistical analyses.

All authors wrote the paper, read, and approved the submitted version.

\section{Data Availability Statement}

The data used to support the findings of this study are available from the corresponding author upon request.

\section{Conflict of interest}

The authors declare that they have no conflict of interest.

\section{Provenance and peer review}

This article has undergone peer review.

\section{Ethical statement}

The study did not warrant institutional review board approval as no human subjects were involved.

\section{Funding}

Supported by the Fundamental Research Funds for the Central Universities(Grant no. 2019jbkyzy002,lzujbky-2019-ct02,2020jbkyzx001,lzujbky-2020-kb20)

Fig.1 Flow diagram;

Fig.2a, b Risk of bias

Fig.3 Association between various phytoestrogens with the effectiveness of depression. 
e-Fig 1. The result of sensitivity analysis

e-Fig.2 The trim and -fill funnel plot.

e-Fig.3 Association between isoflavone and lignans groups with the effectiveness of menopausal women to remission depression in different evaluation scales

e-Fig.4 Association between three intake doses' group with the effectiveness of menopausal women to remission depression.

e-Fig. 5 Association between various scales with the effectiveness of menopausal women to remission depression.

e-Fig.6 Association between isoflavone and lignans groups with the effectiveness of menopausal women to remission depression in different evaluation scales except for KMI and GCS scales.

e-Fig.7 Association between three intake doses 'groups with the effectiveness of menopausal women to remission depression in different evaluation scales except for KMI and GCS scales.

e-Fig.8 Association between eight countries with the effectiveness of menopausal women to remission depression.

Table1. Characteristic of the studies included in the meta-analysis

Table2. The adverse events in all included studies

Reference

1. Glisic M, Kastrati N, Musa J, et al. Phytoestrogen supplementation and body composition in postmenopausal women: A systematic review and meta-analysis of randomized controlled trials. Maturitas.2018;115:74-83.

2. Aghamiri V, Mirghafourvand M, Mohammad-Alizadeh-Charandabi S, Nazemiyeh H. The effect of Hop (Humulus lupulus L.) on early menopausal symptoms and hot flashes: A randomized placebo-controlled trial.Complement Ther Clin Pract. 2016;23:130-135.

3. Gerbarg PL, Brown RP. Pause menopause with Rhodiola rosea, a natural selective estrogen receptor modulator. Phytomedicine : international journal of phytotherapy and phytopharmacology.2016;23(7):763769.

4. Hale G, Bievre M, Hughes C. Exploring the role of progestins and phytoestrogens in menopause. Integrative Medicine.1999;2(2-3):133-141.

5. Llaneza P, García-Portilla MP, Llaneza-Suárez D, Armott B, Pérez-López FR. Depressive disorders and the menopause transition. Maturitas. 2012;71(2):120-130.

6. Perez-Lopez FR, Perez-Roncero G, Fernandez-Inarrea J, et al. Resilience, depressed mood, and menopausal symptoms in postmenopausal women. Menopause (New York, NY). 2014;21(2):159-164.

7. Johnston E, Johnson S, McLeod P, Johnston M. The relation of body mass index to depressive symptoms. Canadian journal of public health = Revue canadienne de sante publique. 2004;95(3):179-183.

8. Lee JY, Park YK, Cho KH, et al. Suicidal ideation among postmenopausal women on hormone replacement therapy: The Korean National Health and Nutrition Examination Survey (KNHANES V) from 2010 to 2012. Journal of affective disorders. 2016;189:214-219.

9. Birkhäuser M. Menopause, oestrogen deficiency and depression.Archives of Women's Mental Health. 2011;14:S97.

10. Faubion SS, Litin SC, Bundrick JB. Clinical Pearls in women's health. Disease-a-Month. 2017;63(6):149157. 
11. Finnell JE, Lombard CM, Moffitt CM, et al. The role of estradiol on social stress induced depressive-like behavior and neuroinflammation in females. FASEB Journal. 2017;31(1).

12. Al-Safi ZA, Santoro N. Menopausal hormone therapy and menopausal symptoms. Fertility and Sterility. 2014;101(4):905-915.

13. Morrison MF, Kallan MJ, Ten Have T, Katz I, Tweedy K, Battistini M. Lack of efficacy of estradiol for depression in postmenopausal women: a randomized, controlled trial. Biol Psychiatry.2004;55(4):406-412.

14. Bai F, Ling J, Esoimeme G, et al. A systematic review of questionnaires about patient's values and preferences in clinical practice guidelines. Patient preference and adherence.2018;12:2309-2323.

15. Lee HW, Jun JH, Kil KJ, Ko BS, Lee CH, Lee MS. Herbal medicine (Danggui Shaoyao San) for treating primary dysmenorrhea: A systematic review and meta-analysis of randomized controlled trials. Maturitas. 2016;85:19-26.

16. Atteritano M, Mazzaferro S, Bitto A, et al. Genistein effects on quality of life and depression symptoms in osteopenic postmenopausal women: a 2-year randomized, double-blind, controlled study. Osteoporosis international. 2014;25(3):1123-1129.

17. Power KA, Thompson LU. Can the combination of flaxseed and its lignans with soy and its isoflavones reduce the growth stimulatory effect of soy and its isoflavones on established breast cancer? Molecular nutrition 83 food research. 2007;51(7):845-856.

18. Lipovac M, Chedraui P, Gruenhut C, Gocan A, Stammler M, Imhof M. Improvement of postmenopausal depressive and anxiety symptoms after treatment with isoflavones derived from red clover extracts. Maturitas. 2010;65(3):258-261.

19. Keshavarz Z, Golezar S, Hajifoghaha M, Alizadeh S. The effect of phytoestrogens on menopause symptoms: A systematic review. Journal of Isfahan Medical School. 2018;36(477):446-459.

20. de Sousa-Munoz RL, Filizola RG. Efficacy of soy isoflavones for depressive symptoms of the climacteric syndrome. Maturitas.2009;63(1):89-93.

21. Su BYW, Tung TH, Chien WH. Effects of phytoestrogens on depressive symptoms in climacteric women: A meta-analysis of randomized controlled trials. Journal of Alternative and Complementary Medicine.2018;24(8):850-851.

22. Norris SL, Meerpohl JJ, Akl EA, et al. The skills and experience of GRADE methodologists can be assessed with a simple tool. J Clin Epidemiol. 2016;79:150-158 e151.

23. Chen Y, Yang K, Marusic A, et al. A Reporting Tool for Practice Guidelines in Health Care: The RIGHT Statement. Ann Intern Med.2017;166(2):128-132.

24. Ge L, Tian JH, Li YN, et al. Association between prospective registration and overall reporting and methodological quality of systematic reviews: a meta-epidemiological study. J Clin Epidemiol. 2018;93:4555.

25. Higgins JP, Thompson SG. Quantifying heterogeneity in a meta-analysis. Statistics in medicine. 2002;21(11):1539-1558.

26. Xiu-xia L, Ya Z, Yao-long C, Ke-hu Y, Zong-jiu Z. The reporting characteristics and methodological quality of Cochrane reviews about health policy research. Health Policy. 2015;119(4):503-510.

27. Flemyng E, Dwan K, Moore TH, Page MJ, Higgins JP. Risk of Bias 2 in Cochrane Reviews: a phased approach for the introduction of new methodology. The Cochrane database of systematic reviews.2020;10:Ed000148.

28. Rosenthal R. The "File Drawer Problem" and Tolerance for Null Results. Psychological Bulletin. 1979;86(3):638-641. 
29. Page MJ, Sterne JAC, Higgins JPT, Egger M. Investigating and dealing with publication bias and other reporting biases in meta-analyses of health research: A review. Research synthesis methods. 2020.

30. Duval S, Tweedie R. Trim and fill: A simple funnel-plot-based method of testing and adjusting for publication bias in meta-analysis.Biometrics. 2000;56(2):455-463.

31. Cheung M, Ho R, Lim Y, Mak A. Conducting a meta-analysis: basics and good practices. International journal of rheumatic diseases.2012;15(2):129-135.

32. Ishiwata N, Melby MK, Mizuno S, Watanabe S. New equol supplement for relieving menopausal symptoms: randomized, placebo-controlled trial of Japanese women. Menopause (New York, NY). 2009;16(1):141148.

33. Hirose A, Terauchi M, Akiyoshi M, Owa Y, Kato K, Kubota T. Low-dose isoflavone aglycone alleviates psychological symptoms of menopause in Japanese women: a randomized, double-blind, placebo-controlled study.Archives of Gynecology and Obstetrics. 2016;293(3):609-615.

34. Park JY, Kim KH. A randomized, double-blind, placebo-controlled trial of Schisandra chinensis for menopausal symptoms. Climacteric : the journal of the International Menopause Society.2016;19(6):574580 .

35. Shamshad Begum S, Jayalakshmi HK, Vidyavathi HG, et al. A Novel Extract of Fenugreek Husk (FenuSMART) Alleviates Postmenopausal Symptoms and Helps to Establish the Hormonal Balance: A Randomized, Double-Blind, Placebo-Controlled Study. Phytotherapy Research.2016;30(11):1775-1784.

36. Evans HM, Howe PRC, Wong RHX. Effects of Resveratrol on Cognitive Performance, Mood and Cerebrovascular Function in Post-Menopausal Women; A 14-Week Randomised Placebo-Controlled Intervention Trial.Nutrients. 2017;9(1).

37. Tice JA, Ettinger B, Ensrud K, Wallace R, Blackwell T, Cummings SR. Phytoestrogen supplements for the treatment of hot flashes: the Isoflavone Clover Extract (ICE) Study: a randomized controlled trial.Jama. 2003;290(2):207-214.

38. Cagnacci A, Arangino S, Renzi A, Zanni AL, Malmusi S, Volpe A. Kava-Kava administration reduces anxiety in perimenopausal women. Maturitas. 2003;44(2):103-109.

39. Bagby RM, Ryder AG, Schuller DR, Marshall MB. The Hamilton Depression Rating Scale: has the gold standard become a lead weight?Am J Psychiatry. 2004;161(12):2163-2177.

40. Kanda F, Oishi K, Sekiguchi K, et al. Characteristics of depression in Parkinson's disease: evaluating with Zung's Self-Rating Depression Scale. Parkinsonism E related disorders. 2008;14(1):19-23.

41. Pollock V, Cho DW, Reker D, Volavka J. Profile of Mood States: the factors and their physiological correlates. The Journal of nervous and mental disease. 1979;167(10):612-614.

42. Travers C, O'Neill SM, King R, Battistutta D, Khoo SK. Greene Climacteric Scale: norms in an Australian population in relation to age and menopausal status. Climacteric : the journal of the International Menopause Society. 2005;8(1):56-62.

43. Alder E. The Blatt-Kupperman menopausal index: a critique.Maturitas. 1998;29(1):19-24.

44. Bu L, Lephart ED. Soy isoflavones modulate the expression of BAD and neuron-specific beta III tubulin in male rat brain. Neurosci Lett. 2005;385(2):153-157.

45. Oxfeldt M, Dalgaard LB, Risikesan J, Johansen FT, Hansen M. Influence of Fermented Red Clover Extract on Skeletal Muscle in Early Postmenopausal Women: A Double-Blinded Cross-Over Study.Nutrients. 2020;12(11).

46. Izumi T, Piskula MK, Osawa S, et al. Soy isoflavone aglycones are absorbed faster and in higher amounts than their glucosides in humans. The Journal of nutrition. 2000;130(7):1695-1699. 
47. Kaari C, Haidar MA, Junior JM, et al. Randomized clinical trial comparing conjugated equine estrogens and isoflavones in postmenopausal women: a pilot study. Maturitas. 2006;53(1):49-58.

48. Davis SR, Briganti EM, Chen RQ, Dalais FS, Bailey M, Burger HG. The effects of Chinese medicinal herbs on postmenopausal vasomotor symptoms of Australian women. A randomised controlled trial. Med $J$ Aust.2001;174(2):68-71.

49. Mason C, de Dieu Tapsoba J, Duggan C, Wang CY, Alfano CM, McTiernan A. Eating behaviors and weight loss outcomes in a 12-month randomized trial of diet and/or exercise intervention in postmenopausal women. The international journal of behavioral nutrition and physical activity. 2019;16(1):113.

50. Yang K. Evidence-based social science: the origin, development and prospects. . Libr Inf. 2018(03):1-10.

\section{Hosted file}

Fig.1. Flow diagram.pdf available at https://authorea.com/users/344317/articles/499912efficacy-and-safety-of-phytoestrogens-in-the-treatment-of-postmenopausal-depressivedisorders-a-systematic-review-and-meta-analysis

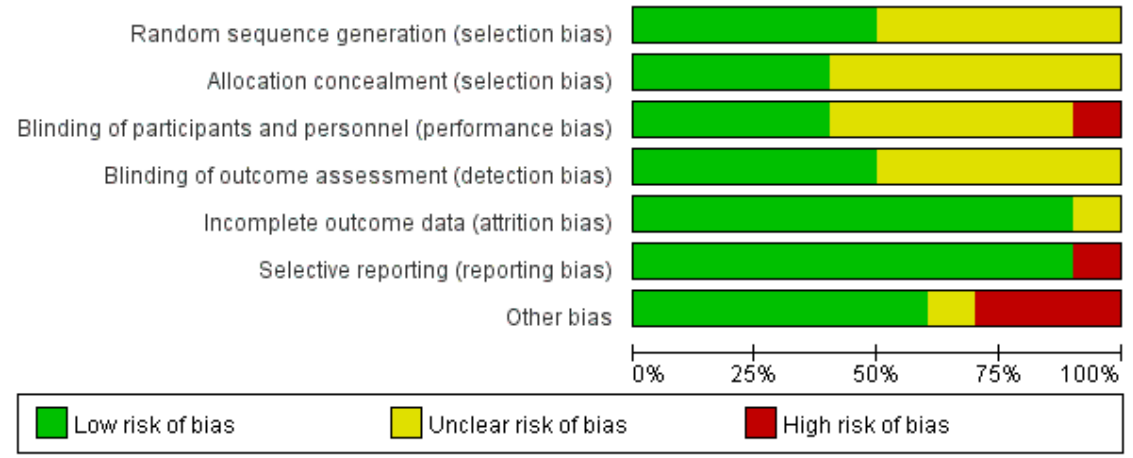




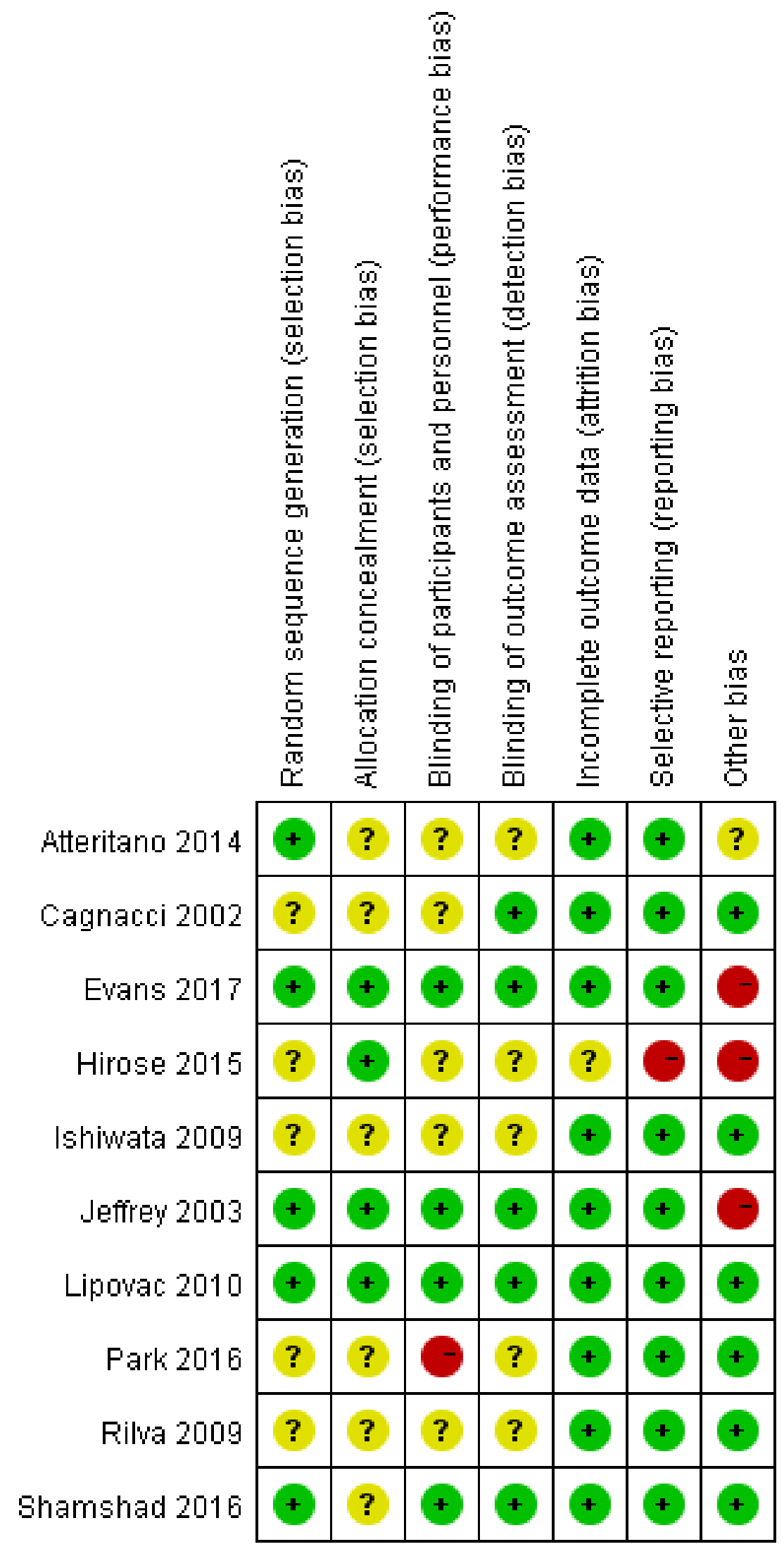




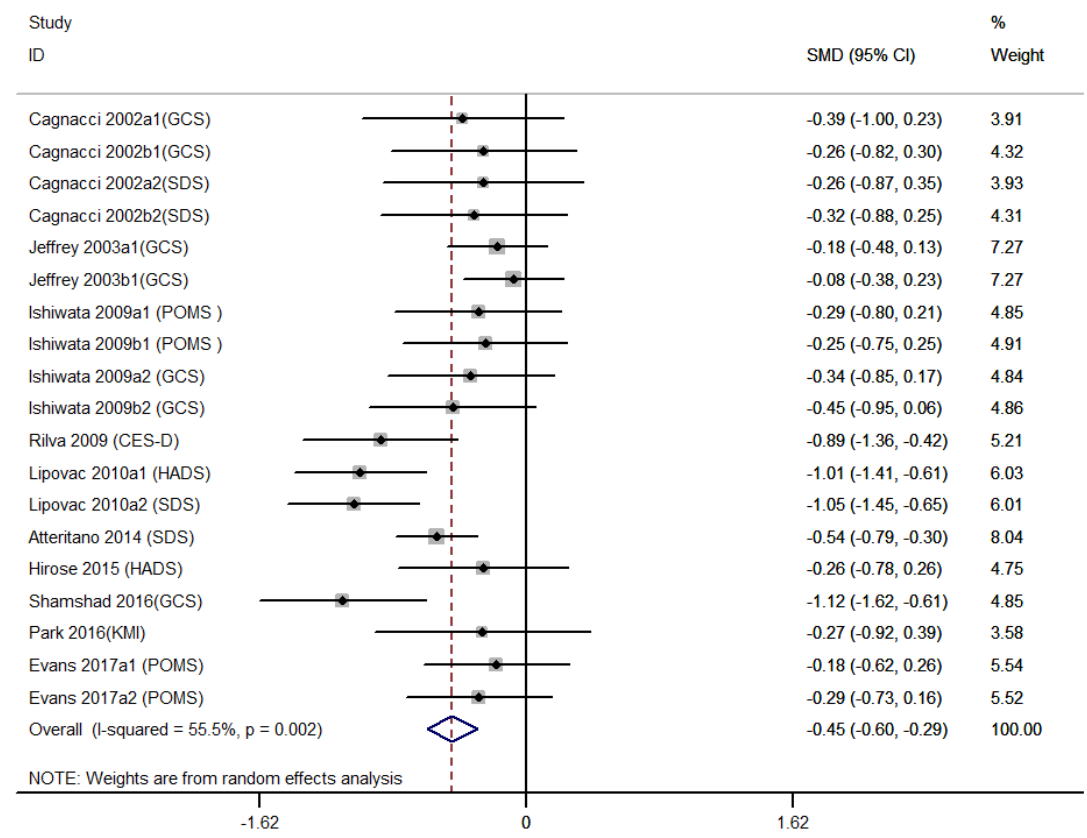

\section{Hosted file}

Table S1.pdf available at https://authorea.com/users/344317/articles/499912-efficacy-andsafety-of-phytoestrogens-in-the-treatment-of-postmenopausal-depressive-disorders-asystematic-review-and-meta-analysis

Sensitivity analysis

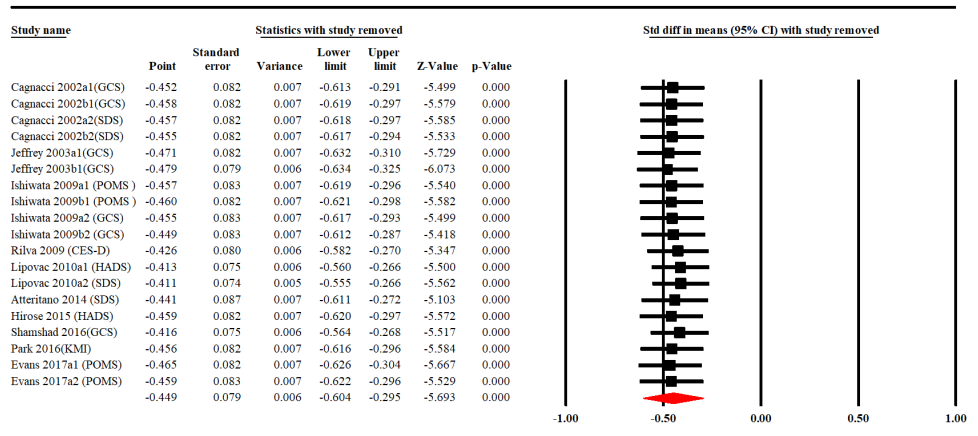



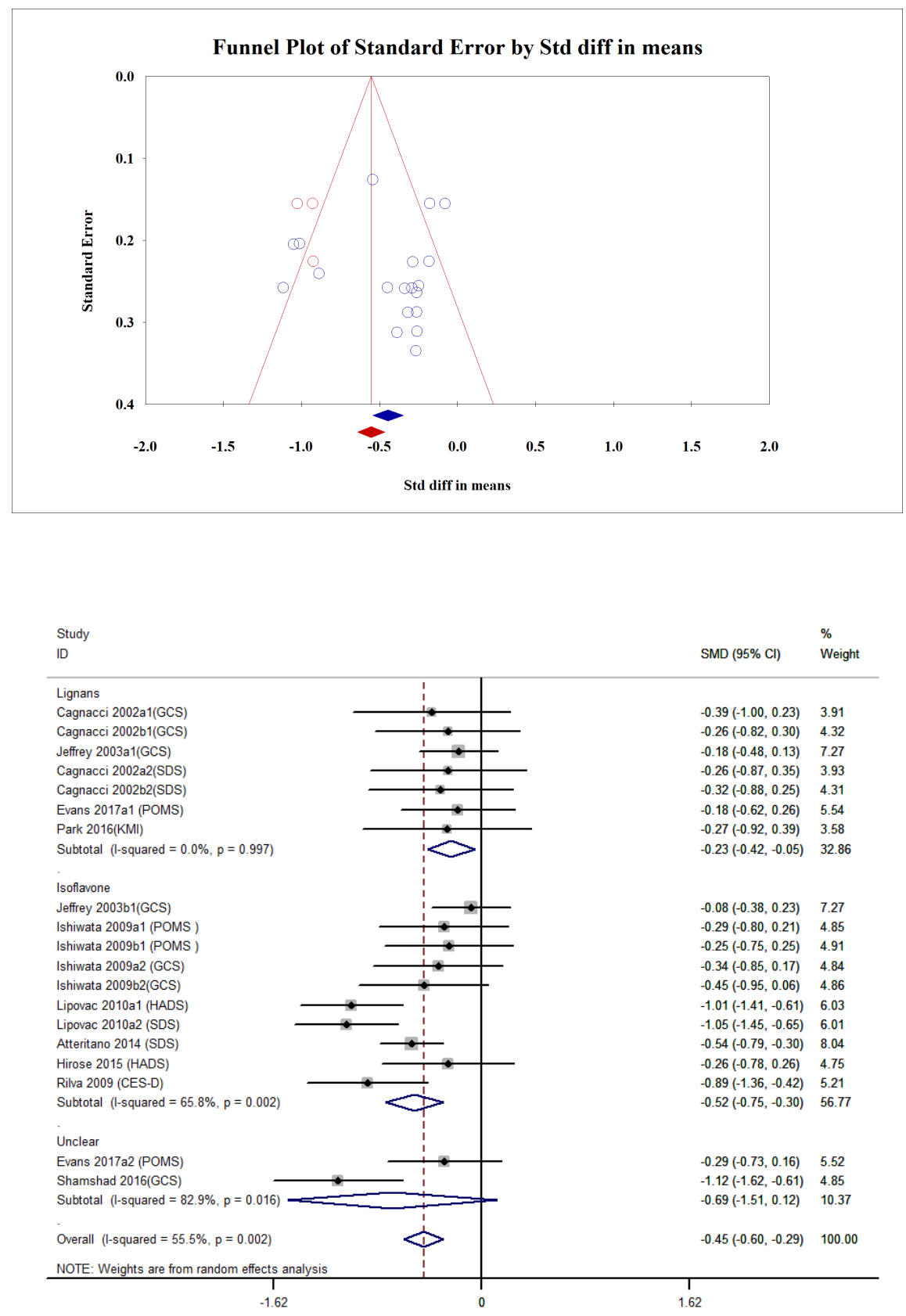


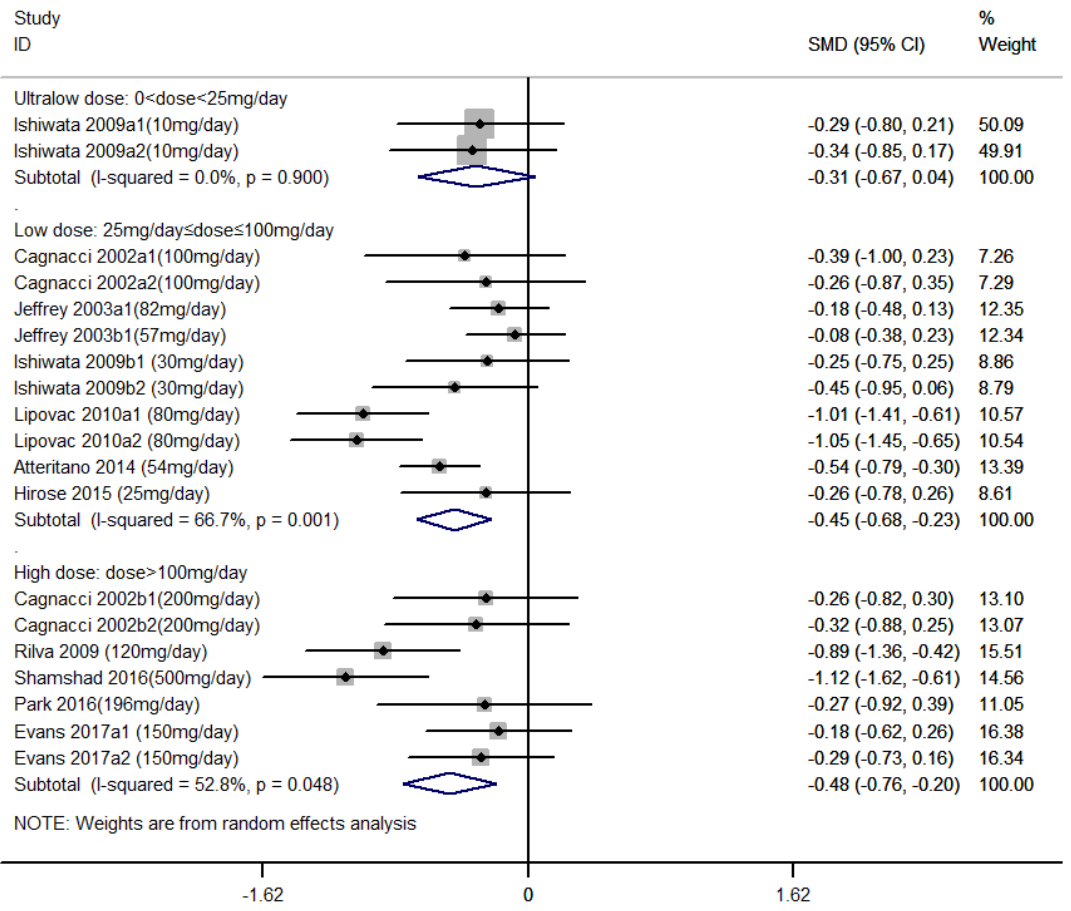




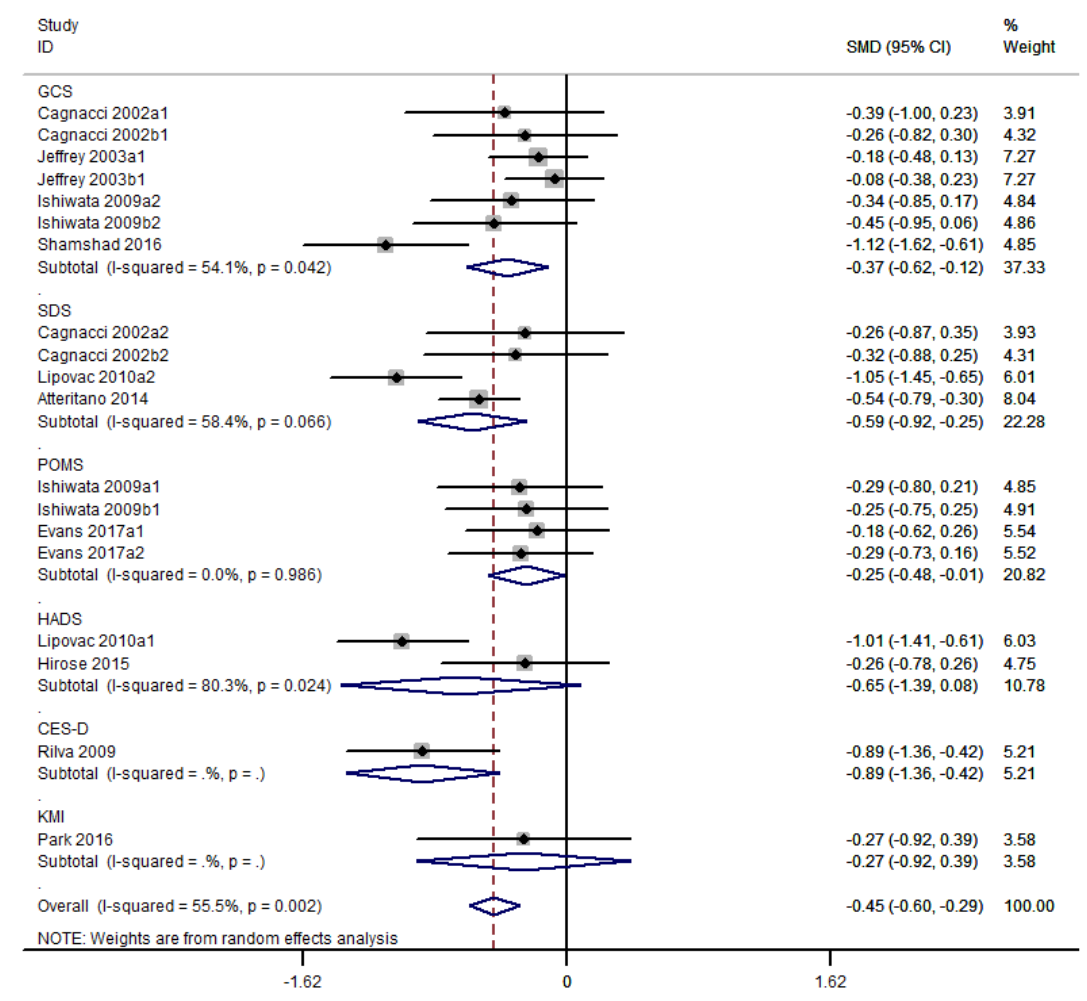

Study
ID
Lignans
Cagnacci 2002a2(SDS)
Cagnacci 2002b2(SDS)
Evans 2017a1 (POMS)
Subtotal (I-Squared = 0.0\%, $\mathrm{p}=0.932$ Cl)




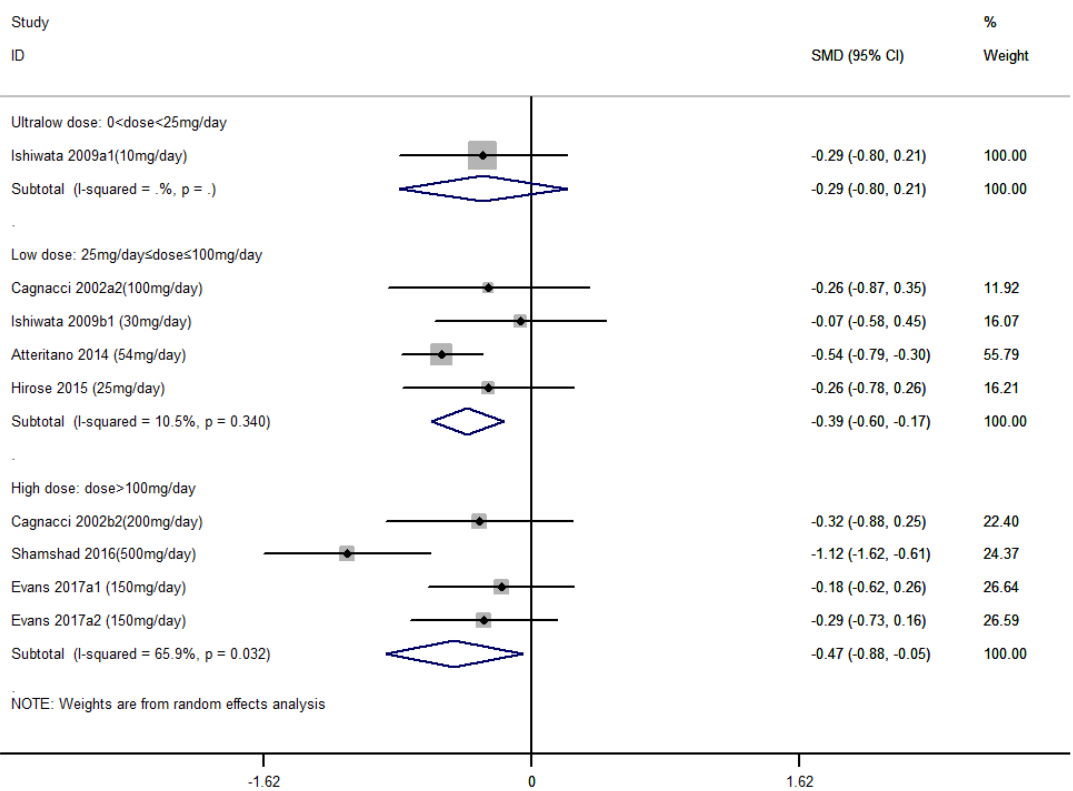

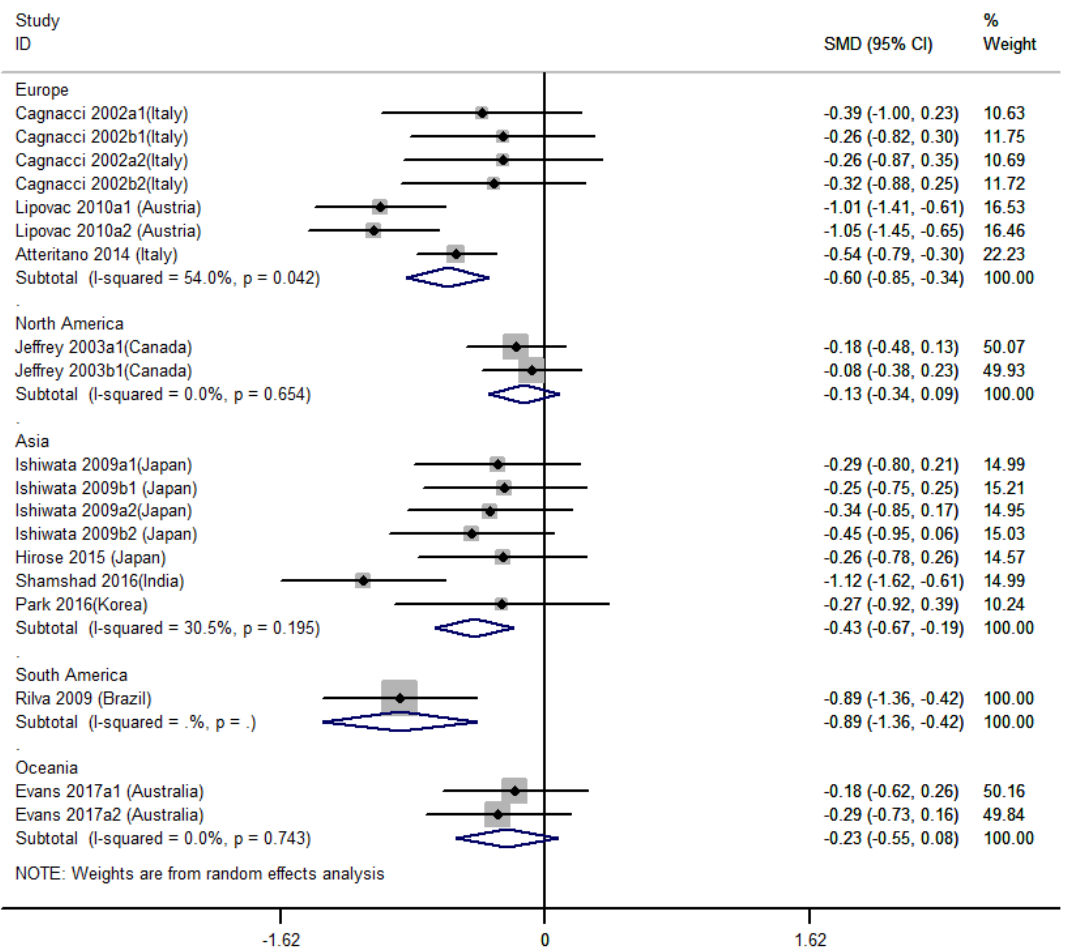

